\title{
Variables psicológicas asociadas con la felicidad
}

\section{Reynaldo Alarcón}

Universidad Nacional Mayor de San Marcos

Lima, Perú

Se reportan los resultados obtenidos en una muestra de estudiantes universitarios peruanos que participaron en una investigación transcultural sobre satisfacción con la vida, concepto que se utiliza en este trabajo como sinónimo de felicidad. Los examinados absolvieron una batería de tests psicológicos de 171 items, que exploraron seis variables presuntamente asociadas a la felicidad. El problema central de este trabajo fue determinar cuál de las variables consignadas en la investigación, predicen "mejor" la felicidad. Se encontró que las variables "satisfacción con la vida" y "frecuencia de afectos positivos" guardan una correlación alta y positiva $(p<.01)$.

felicidad / satisfacción con la vida / afectos positivos

\section{Psychological variables associated with happiness}

The results of a transcultural research in a sample of Peruvian college students regarding life satisfaction are reported. Life satisfaction is a concept used a a synonymous of happiness. The main problem of this research was to determine which of the variables were "better" predictors of happiness. The participants responded a battery of psychological tests of 171 items which explored six variables considered related to happiness. It was found that the variables "life satisfaction" and "frequency of positive affects" are highly and positive correlated $(p<.01)$. 
En la literatura psicológica son frecuentes los trabajos orientados a estudiar comportamientos emocionales que conllevan malestar psicológico de la persona. El énfasis que ha recibido la investigación, por ejemplo, sobre la depresión, la frustración, la desesperanza y la autoestima, obedece, probablemente, a que constituyen desórdenes de la conducta que requieren de intervención psicológica o psiquiátrica. No han recibido la misma atención los comportamientos opuestos a los señalados, que reflejan estados subjetivos como alegría, bienestar, satisfacción con la vida y felicidad. Sin embargo, esta última conducta tiene para el hombre singular valor entre sus metas de vida. La anhela, la busca, se esfuerza por alcanzarla $\mathrm{y}$, a menudo, la idealiza al punto de que la percibe lejana. El eudemonismo consideró la felicidad como el supremo bien que puede alcanzar el hombre.

Se ha sostenido, en efecto, que el hombre busca la felicidad y evita el sufrimiento, entendiendo que la felicidad y el dolor determinan, en cierta manera, la orientación de nuestra conducta. El antecedente más remoto de esta tesis corresponde a Epicuro quien, en su Jardín, que así se denominó su escuela filosófica, enseñaba que el hombre busca la vida dichosa y placentera y que huye de todo lo que le causa dolor, pena y preocupación. El placer que buscaba el epicureísmo no es únicamente el que ofrecen los sentidos, es también de orden espiritual, como el placer afectivo.
La supresión de los estados de desasosiego, de ansiedad, de temor y de dolor, conducen al placer sereno y duradero, material y espiritual, que es la felicidad según Epicuro.

La felicidad puede conceptuarse como aquel estado afectivo de satisfacción plena que experimenta subjetivamente el individuo en posesión de un bien deseado. Esta definición conlleva: a) la felicidad significa sentimientos de satisfacción que vivencia una persona, y sólo ella, en su vida interior; b) el hecho de ser un "estado" de la conducta, alude al carácter temporal de la felicidad, puede ser duradera, pero, a la vez, es perecible; c) la felicidad supone la posesión de un "bien", se es feliz en tanto se posee el bien deseado, la felicidad no tiene sentido sin el bien que hace feliz; d) el bien o bienes que generan felicidad son de naturaleza variada (materiales, éticos, estéticos, psicológicos, religiosos, sociales, etc.), se trata de cosas a las que las personas le asignan cualidades axiológicas, o valencias positivas para decirlo en términos de Lewin. Esto quiere decir que una "cosa" deviene en bien deseado por el valor que le atribuye la persona, de lo cual se infiere que la cosa deseada no posee valor intrínseco y que un mismo bien no siempre genera la felicidad de todas las personas. Observa Blumenfeld (1959) que un mismo objeto puede valorarse desde varios ángulos, dependiendo tanto de la naturaleza del objeto como de la actitud del sujeto, la cual 
tiene su fundamento en la personalidad acuñada por su desarrollo, en su ambiente social y cultural, y en la situación psíquica del momento.

\section{UNA INVESTIGACIÓN TRANSCULTURAL DE LA FELICIDAD}

Ed Diener, del Departamento de Psicología de la Universidad de Illinois, invitó al autor de este artículo a participar en un proyecto de investigación transcultural sobre la felicidad, cuyo significado equivale a los términos en inglés "subjetive well-being", "life satisfaction" o "happiness", que utiliza dicho autor. Se trata de una investigación de múltiples objetivos, que gira en torno a un problema central que busca determinar los factores determinantes de la felicidad, sus componentes y tipos, en las diversas naciones. En la investigación se consideraron variables psicológicas, culturales, educativas, económicas y demográficas. Este megaproyecto involucró a 41 naciones de América, Europa, Asia, África y Oceanía. Los datos se recogieron mediante una batería de tests psicológicos de 171 ítems, que fue absuelta por 7.000 estudiantes universitarios.

Muchos de los resultados de este proyecto, y de estudios precedentes sobre el tema, han sido reportados por Diener y colaboradores. Por ejemplo, Diener, Emmons, Larsen \& Griffin (1985) evaluaron la Escala de satisfacción con la vida; Emmons \& Diener (1985) estudiaron aspectos de la personalidad y el "estar bien"; Larsen, Diener \& Emmons (1985) evaluaron la Escala de estar bien; Emmons \& Diener (1986) estudiaron la influencia de la impulsividad sobre los afectos positivos y negativos; Pavot, Diener \& Fugita (1990) observaron diferencias de género en afectos negativos y felicidad; Diener, Sandvik, Pavot \& Fugita (1992) analizaron la relación entre extraversión y felicidad en Estados Unidos. Asimismo, Diener, Sandvik, Seidlitz \& Diener (1993) estudiaron la relación entre ingreso económico y felicidad; Balatsky \& Diener (1993) investigaron la felicidad en estudiantes rusos; Pavot \& Diener (1993) revisaron la Escala de satisfacción con la vida; y Magnus, Diener, Fugita \& Pavot (1993) estudiaron la extraversión y el neuroticismo como predictores de los eventos de la vida.

El tema de la felicidad ha atraído la atención de otros autores. Bodenhausen, Kramer, \& Suesser (1994) encontraron, a través de cuatro experimentos, que los individuos inducidos a sentirse felices mostraron mayor tendencia a usar estereotipos en juicios sociales, que los del grupo control. Russell \& Wells (1994) examinaron la importancia de 17 predictores potenciales de felicidad matrimonial; señalan, por ejemplo, que el neuroticismo, el número de niños y la relación familiar influyen directamente en la felicidad. Brebner, Donaldson, Kirby, \& Ward (1995) comunican que los factores de personalidad, medidos por el Eysenck Persona- 
lity Questionnaire, están lejos de ser tendencias características de la personalidad; sostienen, también, que la Escala de sociabilidad del Temperament Questionnaire no contribuye a la predicción de la personalidad. Lu \& Shih (1997) hallaron relaciones positivas entre extraversión y felicidad, y entre deseabilidad social y felicidad; de otro lado, comunican relaciones negativas entre neuroticismo, síntomas mentales y felicidad. Cammock, Joseph, \& Lewis (1994) han estimado la confiabilidad de la Escala de Depresión-Felicidad (DHS), encontrando un índice de 0.90; observan que los puntajes altos están asociados con mayor control interno, alta autoestima y bajos rasgos de ansiedad. Furnham \& Chen (1997) administraron el Oxford Happiness Inventory y el NEO Five Factor Inventory a 83 sujetos, y hallaron que la extraversión es un factor positivo de la felicidad. McIntosh, Martin, \& Jones (1997) verificaron la hipótesis de que las personas que creen que su felicidad depende del logro de metas importantes, son influenciadas por pensamientos positivos y eventos negativos de la vida en una magnitud mayor que las personas que no tienen estas creencias. Schyns (1998) ha estudiado la relación entre felicidad y condiciones económicas culturales en 40 países; reporta que ambos factores se correlacionan positiva y fuertemente con la felicidad. Anota que en los países en transición es saludable descubrir una relación causal entre prosperidad económica, cultura y felicidad.

El presente trabajo se enmarca en el proyecto de investigación transcultural sobre la felicidad, antes citado. Se propuso analizar algunos datos obtenidos por la muestra peruana, concretamente se abordan los siguientes problemas: a) verificar las propiedades psicométricas de la Escala de satisfacción con la vida, que fue el instrumento utilizado como medida de la felicidad; b) determinar las relaciones entre las seis variables psicológicas consideradas en la investigación: satisfacción con la vida, frecuencia de afectos positivos, intensidad de experiencias emocionales, extraversión, individualismo y colectivismo; y c) determinar cuál de las variables psicológicas señaladas arriba, tomadas como predictoras, explican "mejor" la felicidad.

\section{MÉTodo}

\section{Participantes}

La muestra estuvo compuesta por 100 estudiantes de universidades privadas de Lima, varones y mujeres, cuyas edades fluctúan entre 18 y 24 años; socialmente se trataba de sujetos de clase media y media alta.

\section{Material}

La prueba utilizada tenía los siguientes subtests: Escala de satisfacción con la vida, Frecuencia de afectos positi- 
vos, Intensidad de experiencias emocionales, Extraversión, Individualismo y Colectivismo. En total, suman 171 los ítems, construidos según la técnica de una escala ordinal, de tipo Likert, aunque para responder los ítems se consideran entre siete y diez categorías.

\section{Procedimiento}

Cada participante recibió la batería de tests reunida en un bloque, una hoja de respuestas y un lápiz. La prueba viene precedida de una instrucción, que informa sobre la finalidad de la investigación, su importancia y la confidencia de las respuestas; además, la instrucción especifica que el examinado no debe escribir su nombre. Las respuestas se marcaron con un lápiz en la hoja respectiva. Aunque no se estableció un tiempo límite para absolver las preguntas, el tiempo promedio empleado fue de 45 minutos.

\section{Resultados}

\section{Verificación de las propiedades psicométricas de la Escala de satisfacción con la vida}

Se consideró necesario verificar las propiedades psicométricas de la Escala de satisfacción con la vida, debido a que, muy a menudo, un test pierde su poder psicométrico original al utilizársele en otra cultura. Se trata de una breve escala integrada por cinco ítems, a los que se responde seleccionando una entre siete alternativas, que van de "completamente de acuerdo" a "completamente en desacuerdo". Los ítems son los siguientes: 5. En la mayoría de las cosas, mi vida está cerca de mi ideal; 6. Las condiciones de mi vida son excelentes; 7. Estoy satisfecho con mi vida; 8 . Hasta ahora, he conseguido las cosas que para mí son importantes en la vida; 9. Si volviese a nacer, no cambiaría casi nada de mi vida.

- Confiabilidad. Se determinó la consistencia interna de la prueba mediante el coeficiente Alfa de Cronbach, obteniéndose $a=.528$, un valor moderado que indica que los ítems del test no covarían en alto grado, como podría esperarse. Utilizando el "método de mitades", corregido por la fórmula de profecía de Spearman-Brown, se obtuvo $r=$ .647 , y según el procedimiento de Guttman split-half $r=.581$. Es probable que el escaso número de ítems de la Escala de satisfacción con la vida afecte su confiabilidad.

En la tabla $\mathrm{N}^{\mathrm{o}} 1$ se reportan las medias, desviación estándar (DS), las correlaciones $(r)$ ítem-test y los coeficientes de determinación múltiples (R2). Puede observarse que el ítem 5 presenta la mayor dispersión de respuestas y que todos los valores de $r$ son altamente significativos, exceptuando el ítem 8, que presenta una correlación no significativa. La media de las correlaciones es $r=.337$ 
Tabla $\mathbf{N}^{\circ} 1$

Medias, desviaciones estándar (DS), correlaciones ítem-test ( $r$ ) y coeficiente de determinación múltiple (R2)

\begin{tabular}{lcccc}
\hline Componentes & $\begin{array}{c}\text { Valores } \\
\text { Eigen }\end{array}$ & $\begin{array}{c}\text { \% de varianza } \\
\text { explicada }\end{array}$ & $\begin{array}{c}\text { Porcentaje } \\
\text { acumulado }\end{array}$ & \\
\hline 5 & 5.060 & 3.196 & $.278^{* *}$ & .1201 \\
6 & 4.550 & 1.258 & $.410^{* * *}$ & .2915 \\
7 & 5.090 & 1.371 & $.336^{* * *}$ & .2850 \\
8 & 4.730 & 1.469 & $.186 \mathrm{~ns}$ & .0391 \\
9 & 4.170 & 1.781 & $.472^{* * *}$ & .2792 \\
\hline
\end{tabular}

${ }^{* *} p<.01 \quad$ *** $p<.001$

$(\mathrm{p}<.001)$. Las correlaciones encontradas indican que los ítems guardan cohesión en alto grado con la escala total. Los coeficientes de determinación múltiple señalan el porcentaje de la varianza explicada por cada ítem de la escala. Los ítems 6, 7 y 9 son los que ofrecen mayores contribuciones, en tanto que el ítem 8 es el que menos aporta a la escala.

- Validez factorial. La validez de constructo de la escala fue evaluada mediante análisis factorial. Se utilizó el Método de componentes principales y la rotación Varimax. La tabla $\mathrm{N}^{\mathrm{o}} 2$ contiene los valores
Eigen de cinco componentes, los porcentajes de la varianza explicada y el porcentaje acumulado de cada componente. Los valores Eigen fluctúan entre 2.018 a 0.499 , observándose que los componentes 1 y 2 presentan valores por arriba de la unidad. Sumados ambos explican el $60.952 \%$ de la varianza total de la escala.

La tabla $\mathrm{N}^{\circ} 3$ presenta dos factores extraídos, rotados por el método Varimax (logrando convergencia en tres interacciones) y las saturaciones factoriales de cada ítem. En primer término, se advierte que la es-

Tabla $\mathbf{N}^{\circ} 2$

Valores Eigen, porcentaje de la varianza explicada y porcentaje acumulado por componente

\begin{tabular}{lccc}
\hline Componentes & $\begin{array}{c}\text { Valores } \\
\text { Eigen }\end{array}$ & $\begin{array}{c}\text { \% de varianza } \\
\text { explicada }\end{array}$ & $\begin{array}{c}\text { Porcentaje } \\
\text { acumulado }\end{array}$ \\
\hline 1 & 2.018 & 40.354 & 40.354 \\
2 & 1.030 & 20.598 & 60.952 \\
3 & .893 & 17.856 & 78.807 \\
4 & .561 & 11.218 & 90.026 \\
5 & .499 & 9.974 & 100.000 \\
\hline
\end{tabular}


cala carece de unidimensionalidad, y que está conformada por dos factores, a los que rehusamos asignarles nombres por tratarse de una muestra relativamente pequeña. Los ítems 7 ("estoy satisfecho con $\mathrm{mi}$ vida"), 6 ("las condiciones de mi vida son excelentes") y 9 ("si volviese a nacer, no cambiaría nada de mi vida") presentan cargas factoriales elevadas y se agrupan en el factor 1 . Estos ítems expresan satisfacción plena con la vida actual y que a la persona no le falta nada. Por el otro lado, el ítem 5 ("en la mayoría de los casos, mi vida está cerca de mi ideal") y el ítem 8 ("hasta ahora he conseguido las cosas que para mí son importantes en la vida"), saturan significativamente al factor 2 . Estos ítems, a diferencia de los del factor 1, reflejan estados temporales de la felicidad, se está satisfecho por lo conseguido hasta la hora presente, pero aún no se ha alcanzado la felicidad plena. El ítem 9 carga también en el factor 2, con .314, un valor no despreciable, ya que a menudo se consideran satisfactorias cargas factoriales mayores o iguales a 0.30. En suma: el análisis factorial ha revelado la composición bifactorial de la escala, y, a la vez, ha verificado su validez de constructo.
Tabla $N^{\circ} 3$

Matriz de factores extraídos por rotación Varimax y cargas factoriales de los ítems

\begin{tabular}{lrcc}
\hline & & Factores & \\
Ítems & 1 & & 2 \\
\hline 7 & .813 & \\
6 & .813 & \\
9 & .701 & .314 \\
5 & .164 & .754 \\
8 & & .732 \\
\hline
\end{tabular}

\section{Correlaciones entre la variable "satisfacción con la vida" y cinco variables psicológicas}

Hemos correlacionado las seis variables psicológicas consideradas en este estudio: "satisfacción con la vida" (SV), "frecuencia de afectos positivos" (FAP), "intensidad de experiencias emocionales" (IEE), "extraversión (E) "individualismo (I) y "colectivismo" (C). Puede advertirse en la tabla $\mathrm{N}^{\circ} 4$ que la variable "satisfacción con la vida" muestra una correlación significativa al .01 de probabilidad con la variable "frecuencia de afectos positivos", ambas conductas guardan estrecha asociación. Sin embargo, la correlación con "intensidad de experiencias emocionales" es negativa, sugiriendo que las emociones intensas o muy fuertes no favorecen la felicidad. Lo propio puede decirse de la correlación entre las variables "satisfacción con la vida" e "individualismo", esta variable se refiere a conductas independientes, egoístas, competitivas y a la búsqueda 
de éxito personal. Otras correlaciones importantes corresponden a "frecuencia de afectos positivos" con "extraversión", probablemente los individuos extravertidos son propensos a manifestar con facilidad sus afectos; asimismo, es significativa la relación entre "frecuencia de afectos positivos" e "intensidad de experiencias emocionales".

\section{Variables psicológicas que explican la "satisfacción con la vida"}

Para determinar cuál o cuáles de las variables psicológicas involucradas predicen "mejor" la satisfacción con la vida, hemos analizado los datos mediante regresión múltiple. En la ecuación de regresión, la variable "satisfacción con la vida" funge de variable independiente y las cinco variables psicológicas, tomadas en conjunto, actúan como variables predictoras. Los resultados del análisis de varianza de la regresión $(\mathrm{F}=4.786$, significativo a $\mathrm{p}<$ .001 ), indican que el coeficiente $\mathrm{R}=$
.450 expresa una relación altamente significativa entre la variable "satisfacción con la vida" y las cinco variables predictoras. El coeficiente de determinación múltiple $\mathrm{R} 2=.203$ señala que las variables predictoras explican el $20 \%$ de la varianza de la variable dependiente. El 80\% restante de variabilidad podrían explicarlo factores no considerados en el presente artículo.

Cuando evaluamos los coeficientes de regresión múltiple para cada variable predictora (tabla $\mathrm{N}^{0}$ 5), encontramos que el único coeficiente de regresión estadísticamente significativo es "frecuencia de afectos positivos". Esta variable explica por sí sola el $16.5 \%$ de "satisfacción con la vida". La razón $\mathrm{F}=$ $19.330(\mathrm{p}<.001)$ indica una relación altamente significativa entre ambas variables. El aporte de las demás variables carece de significación estadística, tal como lo señalan los bajos coeficientes de determinación múltiple; en otras palabras, carecen de valor predictivo.

Tabla $\mathbf{N}^{\circ} 4$

Coeficientes de correlación de Pearson entre las variables psicológicas consideradas en el estudio de la felicidad

\begin{tabular}{lllllll}
\hline Variables & SV & FAP & IEE & E & I & C \\
\hline SV & & $.406^{\star *}$ & -.088 & .100 & -.049 & .010 \\
FAP & $.406^{* * *}$ & & $.219^{*}$ & $.346^{* *}$ & -.084 & -.054 \\
IEE & -.088 & $.219^{\star}$ & & .003 & -.055 & -.108 \\
E & .100 & $.346^{\star *}$ & .003 & & .127 & .140 \\
I & -.049 & -.084 & -.055 & .127 & & $.656^{* * *}$ \\
C & .010 & -.010 & -.108 & .140 & $.656^{* *}$ & \\
\hline
\end{tabular}

${ }^{*} \mathrm{P}<.05$

${ }^{* *} \mathrm{P}<.01$ 
Tabla No 5

Coeficientes de regresión múltiple (R), coeficientes de determinación múltiple (R2), errores estándar de las estimaciones (ES), razones F y coeficientes "beta" estandarizados, para cada variable predictora. Variable dependiente: "satisfacción con la vida"

\begin{tabular}{lccrrr}
\hline Variables & FAP & IEE & E & I & C \\
\hline R & .406 & .088 & -.100 & .049 & .010 \\
R2 & .165 & .008 & .010 & .002 & -.000 \\
ES & 5.126 & 5.587 & 5.581 & 5.602 & 5.609 \\
F & $19.330^{* *}$ & .770 & .993 & .233 & .010 \\
"beta" & .406 & -.088 & .100 & -.049 & .010 \\
\hline${ }^{* * P}<.01$ & & & & &
\end{tabular}

Lo mismo puede afirmarse de la lectura de los coeficientes "beta" estandarizados: la variable FAP tiene el peso relativo más elevado entre las cinco variables predictoras. La variable "intensidad de experiencias emocionales", con signo negativo, estaría indicando que la fuerza de las emociones no contribuye a la felicidad, como se señaló más arriba. Algo similar se puede decir de la variable "individualismo".

Es de presumir que las personas con altas puntuaciones en la Escala de satisfacción con la vida obtengan altas puntuaciones en la Escala de frecuencia de afectos positivos. De la misma manera, los individuos de bajas puntuaciones en SV obtendrán bajas pun- tuaciones en FAP. Para despejar esta suposición se han tomado las puntuaciones ubicadas en el cuartil superior (Q3) y en el cuartil inferior (Q1) de la distribución de "satisfacción con la vida". Se calcularon las medias de cada variable y se les comparó utilizando " $t$ " de Student. Los resultados encontrados (véase tabla $\mathrm{N}^{\mathrm{o}}$ 6) muestran la correspondencia entre las medias de SV y FAP del grupo Q3; vale decir, a mayor satisfacción con la vida corresponde alta frecuencia de afectos positivos. Por otro lado, también se observa correspondencia entre las medias de las variables SV y FAP del grupo Q1. Los valores de " $\mathrm{t}$ " altamente significativos, corroboran la hipótesis propuesta: los

\section{Tabla $N^{\circ} 6$}

Valores " $t$ " de Student al comparar las medias (M) de las puntuaciones de los grupos Q3 y Q1, correspondientes a las variables "satisfacción con la vida" y "frecuencia de afectos positivos"

\begin{tabular}{|c|c|c|c|c|}
\hline Variables & & Grupo Q3 & Grupo Q1 & "t" \\
\hline SV & $M$ & 30.666 & 17.041 & $17.56^{* \star \star}$ \\
\hline FAP & $M$ & 37.560 & 31.08 & 4.24 \\
\hline
\end{tabular}

${ }^{\star * *} p<.001$ ( dos colas) 
afectos positivos contribuyen a la felicidad de las personas.

\section{DISCUSIÓN}

La consistencia interna de la Escala de satisfacción con la vida fue analizada con el coeficiente Alfa de Cronbach y el "método de mitades", corrigiéndose las correlaciónes obtenidas con las fórmulas de Spearman- Brown y de Guttman. Los valores hallados con estos procedimientos revelaron una moderada consistencia interna del instrumento. En tanto, las correlaciones "ítems-test", arrojaron altos coeficientes de correlación. Estos resultados, en cierto modo, concuerdan con los encontrados por Diener, quien asegura que la Escala de satisfacción con la vida tiene buena consistencia interna, confiabilidad temporal y validez (Diener, comunicación epistolar; Pavot \& Diener, 1993). Es probable que de aumentar el número de ítems mejore la confiabilidad de la escala.

La validez de constructo fue determinada por análisis factorial, el mismo que reveló la composición bifactorial de la escala. Era de esperar que si el instrumento estaba destinado a medir un rasgo unitario, sus ítems se agruparan en un solo factor. Sin embargo, teóricamente, si el constructo es complejo, puede presentar más de un componente. Parece que éste es el caso del constructo de satisfacción con la vida y de la escala que lo mide, aunque sería deseable desarrollar un número mayor de ítems para medir con mayor precisión cada factor. En suma: los resultados del análisis factorial pueden tomarse como evidencias de la validez de constructo de la escala, que mide dos dimensiones de la felicidad.

Hemos encontrado que la variable "satisfacción con la vida", guarda una correlación altamente significativa con la variable "frecuencia de afectos positivos"; su correlación con las demás variables es muy baja y en algunos casos los nexos son negativos. También se halló una correlación altamente significativa entre las variables "frecuencia de afectos positivos" y "extraversión". Similar hallazgo ha encontrado Diener en países del Oeste, y se pregunta si la alta correlación positiva entre afectos positivos y extraversión es un fenómeno del Oeste o es universal.

El último problema, y central de este trabajo, fue indagar por las variables psicológicas involucradas en este estudio, que predicen "mejor" la satisfacción con la vida. El análisis de regresión múltiple logró determinar que la "frecuencia de afectos positivos" explica, por sí sola, el 16.5 de la varianza de la variable "satisfacción con la vida". Esto quiere significar que la reiteración de afectos positivos actúan como contingencias que contribuyen a la felicidad de la gente. Queda pendiente identificar qué otros factores psicológicos, y de otros géneros, incentivan la satisfacción con la vida, el estar feliz o simplemente la felicidad. 


\section{REFERENCIAS}

Balastky, G. \& Diener, E. (1993). Subjective well-being among Russian students. Social Indicator Research, 28, 225-243.

Blumenfeld, W. (1959). Valor y valoración. Lima: Universidad Nacional Mayor de San Marcos.

Bodenhausen, G.V., Kramer, G.P. \& Suesser, K. (1994). Happiness and stereotipic thinking in social judgment. Journal of Personality and Social Psychology, 66, 621-632.

Brebner, J., Donaldson, J., Kirby, N. \& Ward, L. (1995). Relationships between happiness and personality. Personality and Individual Differences, 19, 251-158.

Cammock, T., Joseph, E. \& Lewis, C. (1994). Personality correlates of scores on the depression. Psychological Report, 75, 1649-1650.

Diener, E., Emmons, R.A., Larsen, R.J., \& Griffin, S. (1985). The satisfaction with life scale. Journal of Personality Assessment, 49, 71-75.

Diener, E., Sandvik, E., Pavot, W., \& Fugita, F. (1992). Extraversión and subjective well-being in U.S. national probability sample. Journal of Research in Personality, 26, 205-215.

Diener, E., Sandvik, E., Seidlistz, L., \& Diener, M. (1993). The relationship between income and subjective wellbeing: Relative or absolute? Social Indicators Research, 28, 195-233.

Emmons, R.A., \& Diener, E. (1985). Personality correlates of subjetive wellbeing. Personality and Social Psychology Bulletin, 11, 89-97.
Emmons, R.A. \& Diener, E. (1986). Influence of impulsivity and sociability on positive and negative affect. Journal of Personality and Social Psychology, 50, 1211-1215.

Furnham, A. \& Chen, H. (1997). Personality and happinnes. Psychological Report, 80, 761-762.

Larsen, R.J., Diener, E., \& Emmons, R.A. (1985). An evaluation of subjective well-being measures. Social Indicators Research, 17, 1-18.

Lu, L. \& Shinh, J.B. (1997). Personality and happiness: Is mental health a mediator? Personality and Individual Differences, 22, 249-256.

Magnus, K., Diener, E., Fugita, F., \& Pavot, W. (1993). Extraversion and neuroticism as predictors of objective life events: A longitudinal analysis. Journal of Personality and Social Psychology, 65, 1046-1053.

McIntosh, W.D., Martin, L.L. \& Jones, J. B. (1997). Goal beliefs, live events, and the malleability of people's judgments of their happiness. Journal of Social Behavior and Personality, 12, 567-575.

Pavot, W., Diener, E., \& Fugita, F. (1990). Extraversión and happiness. Personality and Individual Differences, 11, 12991306.

Pavot, W., \& Diener, E. (1993). Review of the satisfaction with life scale. Psychological Assessment, 5, 164-172.

Russell, R.J., \& Wells, P.A. (1994). Predictors of happiness in married couples. Personality and Individual Differences, 17, 313-321.

Schyns, P. (1998). Crossnational differences in happiness: economic and cultural factors explored. Social Indicators Research, 43, 3-26. 


\section{La explicación en psicología: hacia una teoría causal singular*}

\section{Ricardo Braun}

Universidad de Lima

Lima, Perú

El problema de la explicación científica en general y de la psicológica en especial es analizado desde el punto de vista filosófico. La tesis fundamental es que la causalidad es un componente necesario de la explicación. Se revisa la influencia del modelo hempeliano de explicación científica dentro de la psicología y se afirma que bajo este modelo la ciencia psicológica podría ser descartada. Se contempla el análisis de un modelo alternativo que no contiene el componente causal, lo cual el autor considera inadecuado. Finalmente, se esboza una propuesta de una teoría causal singular de la explicación como alternativa para la explicación psicológica.

explicación / singularidad / regularidad / ley

\section{Explanation in psychology: towards a singular causal theory}

The problem of scientific explanation in general and in psychology in particular is analized from a philosophical standpoint. The central thesis is that causality is a necessary component of explanations. The impact of the hempelian model on psychology is revisited and the author suggests that under that model, psychology as a science is discarted. A non-causal model of explanation is discussed and shown to be inadequate as a form of explanation. Finally, a theory of singular causation is suggested as a model for psychological explanations.

* Investigación auspiciada por el Instituto de Investigación Científica de la Universidad de Lima Dirección del autor: Rbraun@correo.ulima.edu.pe 
En los últimos años ha recobrado nuevas fuerzas la investigación acerca de la situación de las explicaciones de la psicología popular como una forma de explicación científica. A pesar de la impresión que provocaron los ataques feroces sobre la psicología popular, el análisis serio del destino de la psicología popular por parte de filósofos y psicólogos es relativamente un nuevo fenómeno. Por varios años la filosofía de la mente tenía poco que decir acerca de los estados psicológicos intencionales tales como creencias, deseos, emociones, motivos, etc. ${ }^{1}$. Básicamente, las discusiones estuvieron restringidas a la reducción de los aspectos cualitativos de sensaciones, como el dolor, a estados cerebrales a través de la influyente teoría de la identidad; en otras palabras, traduciendo el mundo de lo intencional a algo más empíricamente acep-

1 El concepto de intencionalidad fue utilizado en el medioevo y tomado posteriormente en el siglo XIX por Franz Brentano. En el sentido de Brentano el concepto se refiere a la propiedad de la mente de estar dirigida hacia o sobre objetos y estados en el mundo. Continuando en esta tradición, la filosofía analítica contemporánea considera la intencionalidad como la serie de fenómenos mentales asociados con los términos creencia, deseo, intención, temor, amor, odio, disgusto, emoción, memoria, percepción y la acción intencional. No debe confundirse la palabra técnica intencional con la palabra intencional usada popularmente para referirse a realizar una acción "con intención”. En todo caso, esa sería una forma más de intencionalidad, sumada a las anteriores mencionadas. Para una explicación introductoria ver Guttenplan (1995, pp. 379ss.). table. Poco aporte podía brindar la filosofía de la mente al psicólogo practicante puesto que, en otro frente, los estados psicológicos intencionales eran negados con el dominio de las diferentes formas de conductismo. Los únicos análisis filosóficos que se consideraban relevantes para la psicología eran las justificaciones del conductismo lógico. Por lo tanto, había muy poco análisis filosófico que propiamente pudiera llamarse filosofía de la psicología, esto es, una disciplina específicamente preocupada con los estados psicológicos teoréticos postulados por la psicología.

Hoy, sin embargo, existe tal dominio, $\mathrm{y}$ ha venido con un aire de revancha. La razón es la revolución cognitiva en psicología que ha creado una empresa multidisciplinaria conocida como la "ciencia cognitiva"2. La aproximación cognitiva parecía condenada al fracaso porque dependía de la introspección de los estados y procesos psicológicos de los sujetos experimentales. Sin embargo, los substanciales logros teoréticos de la psicología cognitiva han demostrado la falsedad de estas sospechas iniciales.

La filosofía de la mente se ha movido en una dirección similar y sus intereses han pasado de los fenómenos de sensaciones a los fenómenos psicológicos intencionales tales como creencias y deseos. Los famosos trabajos de Do-

2 Una de las mejores introducciones es la colección en cuatro volúmenes de Osherson (1995). 
nald Davidson sobre la explicación de la acción humana (agente) resultaron en una actitud de respeto a las explicaciones derivadas de la psicología popular como una forma de explicación causal que podría jugar un rol importante en la psicología científica. Los fenómenos intencionales estarían siendo tratados como entidades teoréticas y, por lo menos, ameritaría un análisis en términos de su metafísica y rol causal.

Es probablemente en los trabajos de Jerry Fodor en los que filósofos y psicólogos han encontrado un campo común para un desarrollo conjunto. En efecto, en su conocida obra The language of thought (1975), Fodor expone el compromiso ontológico de cualquier ciencia cognitiva futura, a saber, el realismo intencional, la doctrina que considera que las proposiciones actitudinales (propositional attitudes) son estados con contenido (son semánticamente evaluables) y causalmente eficaces (funcionales) instanciados en sistemas neuronales.

Por supuesto, no todos los filósofos han sido particularmente caritativos con la psicología popular. Como ocurre en cualquier argumento filosófico posible, siempre encontramos juntos amigos y enemigos. En el lado adversario están aquéllos que consideran que nuestras explicaciones psicológicas populares terminarán siendo inexactas e inferiores a explicaciones alternativas teoréticas del comportamiento humano en términos de procesos neurofisioló- gicos. Si éste resultara ser el caso, se sostiene que deberemos abandonar nuestras tan amadas formas de explicación psicológica popular y, con ello, la ontología de los estados psicológicos con contenido que ella presupone. Más aún, se argumenta que las entidades postuladas en nuestras mejores teorías de la ciencia cognitiva no podrán ser, de ninguna manera, identificables con las postuladas en las explicaciones de la psicología popular. Por lo tanto, nos veremos forzados a concluir que no hay entidades que tengan propiedades esenciales asociadas a los fenómenos que se asocian con la psicología popular. Adicionalmente, las entidades usadas para la descripción de los estados en la psicología popular no pertenecen a la clase que permite sostener leyes causales, tal como encontramos en las ciencias físicas, y por lo tanto, o bien la psicología se mantiene discontinua con respecto a las demás ciencias y descalificada como una disciplina científica, o bien, nuestras entidades psicológicas populares serán abandonadas.

Estos juicios acerca de la psicología popular suponen una visión particular sobre la naturaleza de la explicación científica y el tipo de entidades que debiera contar como tipos naturales para los propósitos de la explicación. Sin embargo, las posiciones acerca de aquello que pueda ser considerado como explicación científica es todavía controversial, y por lo tanto requiere de mayor análisis. 
En lo que sigue, resumiré algunos argumentos y tesis en discusión actual. Mi propósito es, en último término, demostrar que las entidades postuladas por la psicología popular son adecuadas para la explicación y que, por lo tanto, juegan un rol importante en la psicología científica. No abordaré esa conclusión en esta oportunidad. Más bien, en este artículo quiero señalar que supuestos impedimentos y objeciones a la tesis que defiendo pueden ser superados. Éste es el plan: primero, examinaré la naturaleza de la explicación científica. Argumentaré que el modelo dominante, a pesar de sus modificaciones, tiene serias dificultades y no supone un impedimento para otros modelos alternativos. Segundo, examinaré la aseveración que la acción humana debería ser estudiada bajo un modelo no-causal. Es posible rebatir esta idea mostrando que el causalismo es compatible con la comprensión de la acción humana como una actividad significativa. Finalmente, esbozaré algunas ideas de la propuesta de un modelo de explicación científica, al menos para algunos fenómenos. Escojo estos temas porque considero que pueden ser interesantes para la comunidad psicológica.

\section{LA NATURALEZA DE LA EXPLICACIÓN CIENTÍFICA}

Es importante introducir algunos temas de la explicación científica que posteriormente serán relevantes en nuestra discusión. Algunas de las pre- guntas que necesitamos revisar son: ¿Qué es una explicación científica? ¿Cuál es el propósito de una explicación científica? ¿Qué hace que una explicación sea adecuada? ¿Deberíamos estar comprometidos con la unificación de la explicación científica?

Parece claro que uno de los objetivos de la ciencia es la de proveer explicaciones. Aun cuando dar explicaciones es una actividad común en la que se embarcan tanto científicos como la gente ordinaria, tendemos a confiar más en las explicaciones científicas para comprender mejor cómo las cosas ocurren, quizás porque la ciencia es nuestra mejor manera de generar explicaciones que han probado ser exitosas, al menos bajo algunos criterios. Algo sorprendente es que ha habido poco análisis acerca de la naturaleza de la explicación científica, si consideramos el importante impacto que tiene la ciencia en nuestras vidas diarias. La cantidad de literatura existente sobre la explicación científica se mueve alrededor del legado del empirismo lógico, especialmente el trabajo de Hempel (1965) y su modelo "nomológico-deductivo" o de "cobertura legal" (ver más adelante). La filosofía de la explicación científica se ha visto coloreada de una $u$ otra forma por la poderosa influencia de Hempel. Como observa Garfinkel: "es algo escandaloso que se haya prestado poca atención a esta necesidad por la filosofía tradicional que, con pocas excepciones, ha dicho nada acerca de 
las formas de explicación" (1981, p. 19).

La necesidad de un análisis de otras formas de explicación se vuelve evidente cuando una definición de lo que constituye una explicación científica restringe formas alternativas. Consideremos, por ejemplo, cómo la estrategia de investigación en psicología estuvo en el pasado determinada por el modelo de Hempel en los trabajos de psicólogos como Hull y Spence. Luego, otros psicólogos tan diferentes como Maslow y Skinner se unieron para rechazar el modelo hempeliano prevalente (Boden, 1972, p. 32).

La noción de explicación está necesariamente unida a la noción de información. En un sentido ordinario, cuando pedimos una explicación estamos buscando algo de información. Lo mismo se aplica a la explicación científica. Cuando una persona común busca una explicación, claramente la persona quiere comprender por qué una cosa particular ocurre. Una madre preocupada puede preguntar si su hijo/hija tiene fiebre. La madre está preocupada por comprender por qué el evento "fiebre" tiene lugar, es decir, qué lo originó. Nuestra noción intuitiva de explicación está estrechamente ligada con aquélla de causación. La madre en el ejemplo no está buscando una proposición normativa ni un comentario crítico. Presumiblemente, su cuestionamiento busca entender la historia causal de la fiebre. Las explicaciones científicas siguen el mismo patrón. Consideremos, por ejemplo, la explicación de la sorprendente coincidencia de las líneas costeras de Norte y Sudamérica con las líneas costeras de Europa y África. Los geólogos que buscaban una explicación querían comprender el porqué de esta coincidencia. La respuesta a esta inquietud, que se encuentra en el fundamento de la teoría actual del desplazamiento del suelo marino, involucra el dar una historia causal del rompimiento y separación de los continentes que alguna vez estuvieron unidos. Así, nos referimos a la teoría del desplazamiento del suelo marino como una explicación del fenómeno mencionado antes. $Y$ es una explicación porque proporciona un reporte causal del fenómeno.

La correlación entre explicación e historia causal es profundamente intuitiva. Como vimos anteriormente, cuando queremos saber por qué un evento ocurrió, preguntamos qué lo hizo ocurrir. Y lo que normalmente queremos escuchar es algo causal. Child (1994) caracteriza la fundamentación para esta intuición así:

Cada evento o bien tiene una causa o no la tiene. Si tiene una causa, entonces explicar por qué el evento ocurrió tiene que hacer referencia a esa causa. Si no tiene una causa, entonces simplemente no hay explicación de por qué este evento particular ocurrió cuando lo hizo (p. 92).

Tradicionalmente, el enfoque causal a la explicación ha sido asociado al objetivo de la unidad de la ciencia a través 
del reduccionismo. Después de todo, si la realidad está hecha de lo mismo, en último término debería ser explicada en un solo vocabulario, el de la física. Sin embargo, varios problemas aquejan al proyecto reduccionista: primero, no siempre las explicaciones reduccionistas son mejores explicaciones, en el sentido de que no permiten una mayor comprensión del problema y, segundo, contrariamente a un punto defendido por reduccionistas tradicionales, la física teorética es incompatible con el microrreduccionismo.

\section{LA EXPLICACIÓN PSICOLÓGICA BAJO}

\section{EL MODELO NOMOLÓGICO-DEDUCTIVO}

Por un largo período de tiempo, los filósofos de la ciencia creyeron que el modelo de Hempel, conocido posteriormente como el modelo de cobertura legal, era esencialmente correcto. La idea detrás del modelo de cobertura legal es que las explicaciones científicas necesitan leyes (la explicación de un evento particular es "cubierta" por una ley) y que sin leyes no habría una genuina explicación científica. En los años sesenta se había logrado un consenso y aun cuando hubo críticas al modelo, se erigió como una respuesta satisfactoria a uno de los problemas de la filosofía de la ciencia, la explicación científica. Esta actitud puede ser parcialmente explicada por el hecho de que el modelo podía ser formalizado de una manera lógica adecuada y podía, en consecuencia, ser aplicado a las diferentes disciplinas científicas.

El modelo de cobertura legal se convirtió en el marco de referencia desde el que se evaluaban propuestas de explicación. Supuestas explicaciones que no entraban en el modelo eran consideradas pseudoexplicaciones. Como se dijo, implícito en el modelo estaba la creencia de que la explicación científica requería del descubrimiento de leyes. Esto no parecía un problema para las ciencias físicas, donde el número de leyes es más bien abundante, al menos desde la perspectiva tradicional del concepto de ley física. El énfasis en las leyes provocó la construcción de un ideal al que difícilmente las otras ciencias, en particular las humanas/sociales podían acercarse. Como resultado de esto, el programa de desarrollo de la ciencia tenía que buscar el descubrimiento de leyes, si se pretendía continuar en el establishment científico. Así explica Miller (1987) el impulso que guiaba a los primeros científicos sociales:

Los pioneros de la sociología académica moderna, la antropología y la economía, Weber, Durkheim, Radcliffe-Brown, Malikowski, Menger, Jevons, Walras, todos consideraban la subsunción bajo leyes generales como esencial en la explicación científica, y tomaron el descubrimiento de tales leyes como medio para convertir las ciencias sociales en verdaderamente científicas. El compromiso con el modelo de cobertura legal ha mantenido tales objetivos aún frente a continuas decepciones (1987, p. 28). 
En los últimos años, sin embargo, el modelo hempeliano ha sido sujeto de numerosas críticas desde diferentes ángulos y ha habido varios intentos para reformularlo o reemplazarlo. Esta crítica ha creado una inestabilidad en el consenso mencionado, pero esta inestabilidad debería ser bienvenida porque, especialmente, en el caso de las explicaciones en las ciencias sociales, los esfuerzos para adecuar sus explicaciones al modelo estaban condenados al fracaso.

En psicología, la presión para adecuarse al modelo de cobertura legal se ha sentido en los escritos de varios filósofos influyentes. Por ejemplo, en los trabajos de Davidson en la filosofía de la psicología se toma por dada la validez del modelo hempeliano. En efecto, el pronunciado contraste entre las ciencias físicas y sociales es para Davidson resultado de la imposibilidad de las últimas de producir leyes como lo hacen las primeras. En "Psychology as Philosophy", Davidson dice:

... no se puede esperar que las ciencias sociales puedan desarrollarse de manera exactamente paralela a las ciencias físicas, ni podemos esperar que podamos alguna vez explicar y predecir la conducta humana con la misma precisión que es posible en principio para los fenómenos físicos (1980, p. 230).

La conclusión de este enfoque es que eventualmente se llegará a una descalificación de la psicología como empresa científica. Esto, por supuesto, es una consecuencia que se sigue de la aplicación del modelo de cobertura legal. Es interesante notar que no es coincidencia que los escritos a los que se alude fueron publicados en los años sesenta y setenta cuando, como se dijo anteriormente, había un consenso acerca de la naturaleza de la explicación científica.

Recientemente, otros filósofos explícitamente han expresado su adhesión al modelo. Kim, por ejemplo, expresa su visión acerca de las leyes y la psicología así:

La pregunta si debe o no haber leyes psicológicas es una de considerable interés. Puede ser demostrado que si no hay tales leyes, una ciencia psicológica nomotética será imposible. El calificativo "nomotético" es redundante: se supone que la ciencia es nomotética. El descubrimiento, o al menos, la búsqueda de leyes es constitutiva de la misma naturaleza de la ciencia de tal modo que donde no hay leyes, no puede haber ciencia... La ausencia de leyes psicológicas supone la imposibilidad de la psicología como ciencia (1993, p. 194).

Lo que ésta y otras posturas similares tienen en común es la fe inmutable en la validez del modelo de cobertura legal. En esta perspectiva, si la psicología quiere ser bienvenida en las filas de la ciencia, mejor será que se adecue al modelo, y esto significa, descubrir leyes.

Las leyes en psicología, se argumenta, tienen que ser, si no radicalmente diferentes a las de la física, adecuadas a su objeto de estudio. La particularidad del objeto de la explicación, a saber, los eventos mentales, requieren de un tipo diferente de leyes. Horgan y Tienson 
explican por qué algunas características de los eventos mentales demandan un diferente tipo de leyes:

La actividad mental... típicamente involucra una variedad de estados mentales intencionales, todos presentes en el disparador cognitivo al mismo tiempo, ejerciendo simultáneamente influencia causal entre sí y exigiendo simultáneamente atención. Estos estados, individualmente o en combinación, tienen diferentes tendencias a causar ciertos nuevos estados psicológicos y/o conductas, tendencias que están determinadas por la estructura intencional y el contenido (1990, p. 268).

Estas tendencias en competencia provocarán que algunas tendencias sean dominadas por otras y que se haga imposible cualquier generalización del comportamiento. Tratar de describir, a través de leyes psicológicas estrictas, las complejas combinaciones de los estados mentales y el posible resultado de su interacción se convierte en una tarea imposible. El procesamiento mental inevitablemente conlleva a lo que Horgan y Tienson llaman indeterminación psicológica: dada una descripción psicológica de un sistema cognitivo además de todas las leyes psicológicas relevantes, no hay forma de aseverar el resultado de todos los factores psicológicos en competencia (p. 269). Por ejemplo, Verónica lleva un paraguas cuando se va de compras. La explicación, aparte de citar alguna ley, incluirá una descripción de las creencias y deseos de Verónica como condiciones iniciales. Pero, ¿cómo podríamos estable- cer esas condiciones de una manera precisa? Puesto que, por todo lo que sabemos, puede ser que Verónica, quien ama la moda, lleva un paraguas como parte de su traje, llueva o no. O puede ser que Verónica, que necesita un bastón, pero se avergonzaría de usarlo, opta por usar un paraguas. $\mathrm{O}$, de repente, es el caso de que Verónica es supersticiosa y llevando un paraguas mantendrá la mala suerte muy lejos. Los factores en competencia conspiran contra la posibilidad de determinar las condiciones iniciales $\mathrm{y}$, por lo tanto, pone una dificultad para obtener el resultado conductual final.

Estas características sugieren un tratamiento especial de la noción de ley; al menos eso se argumenta. Si las explicaciones dependen de leyes, entonces, el tipo de leyes que se debe buscar es el de leyes ceteris paribus, o las llamadas, leyes "blandas". Las leyes "blandas", sostienen Horgan y Tienson, no son inmunes a la verificación empírica o a la falsación. Por el contrario, describen tendencias causales falsables que pueden ser verificadas por medio de técnicas estadísticas estándar ( $\mathrm{p}$. 270).

Una ley psicológica ceteris paribus tendría la siguiente forma:

(L) Dada una persona $x$, si $x$ desea $p$, y $x$ cree que $m$ es el medio para obtener $p$, ceteris paribus, entonces, $x$ hace $m$.

Supongamos que queremos explicar por qué Eduardo fue al refrigerador para buscar una cerveza. La explicación 
de la acción supondrá usar (L) y todo lo que necesitaríamos para explicar adecuadamente esta acción es "llenar las partes en blanco".

Aquí no me detendré en las críticas formales al modelo de cobertura legal, que podría ser discutido en otra entrega, sino me concentraré en los aspectos pertinentes a la explicación psicológica.

Primero, la aseveración de la existencia de leyes verdaderas y que no tienen excepción está basada más en deseo que en hecho. Es quizás el mismo tipo de esperanza que ha nutrido la creencia de que eventualmente habrá leyes disponibles para todas las explicaciones, al menos en las ciencias físicas. En $A$ brief history of time, Hawking parece endosar esta visión cuando escribe que "existen fundamentos para un optimismo cauto que podemos estar cerca del fin de la búsqueda de las leyes fundamentales de la naturaleza" (1988, p. 156). Puede ser el caso que tengamos más leyes, pero no del tipo que han imaginado los teóricos del modelo hempeliano.

Indudablemente, los científicos son capaces de explicar fenómenos complejos. Pero lo que es admitido menos frecuentemente es que en la mayor parte de los casos, los fenómenos son explicados en la ausencia de leyes de cobertura. Simplemente, la razón es que, contrariamente a la creencia popular, las leyes de cobertura son escasas (Cartwright, 1983, p. 45).
En How the laws of physics lie, Cartwright explica que para efectos prácticos, tomamos las leyes de la física como si no tuvieran excepciones, pero las tienen. Muchas de las leyes que tomamos por "universales" se mantienen sólo para ciertas condiciones ideales que no están normalmente especificadas. Como una ilustración, Cartwright nos presenta la ley de Snell. La ley afirma que un haz de luz cayendo en un plano se transmite al vidrio y se refracta a medida que pasa por la superficie siguiendo una ecuación precisa. Lo que no se especifica en la formulación de la ley es que esta ley es sólo verdadera si el medio es isotrópi$\mathrm{co}^{3}$. Se podría decir que esta ley se aplica en la mayoría de los casos, pero esto ni siquiera es verdad porque la mayoría de los medios son anisotrópicos (Cartwright, 1983, p. 47).

Lo que quedaría sería introducir un modificador a la ley, una cláusula ceteris paribus para indicar que la ley es válida sólo en condiciones especiales. Nótese, sin embargo, que la ley tal como se presenta en su formulación original es simplemente falsa, o como dice Cartwright: "las leyes sin cláusulas $c e$ teris paribus no son sólo falsas, sino consideradas falsas por nosotros"

3 Un medio isotrópico es aquél cuyas propiedades (en este caso ópticas) son las mismas en cualquier dirección que sean medidas. Inversamente, un medio anisotrópico tiene diferentes propiedades ópticas en distintas direcciones. 
(1983, p. 45). Más aún, no existen leyes para condiciones que son inferiores a las ideales. Esto no sólo es verdad para la ley de Snell o similares, sino para la mayoría de las leyes. En este sentido, es posible afirmar que no hay coincidencia entre las leyes y los acontecimientos del mundo: las hojas de los árboles no caen nunca siguiendo la ley de Galileo.

Admitiendo lo anterior, pudiera creerse que, si bien en la física se puede postular la existencia de leyes ceteris paribus, ¿por qué no sugerir el descubrimiento de leyes ceteris paribus en psicología? Después de todo, por qué no imitar a la física en su metodología. Por razones que expondré, aun cuando se sostenga la existencia de leyes ceteris paribus en física, ello no autoriza un tratamiento similar en psicología.

Recordemos la forma sugerida de una ley ceteris paribus en psicología (L). Veremos que es una ley extremadamente vaga en el sentido que no se puede especificar las condiciones de su aplicabilidad. Comparemos (L) con la ley de Snell. Aun cuando sabemos que la ley sólo se aplica a medios isotrópicos (que rara vez ocurre) decidimos usarla en medios anisotrópicos (sabiendo que aunque falsa, pueda ser útil). En contraste, en las leyes psicológicas ceteris paribus, las condiciones, por definición, no pueden ser especificadas. Y si tratáramos de especificarlas, la ley perdería cualquier indicio de generalización, puesto que para especi- ficar las condiciones tendríamos que proporcionar todas las circunstancias individuales para que la ley tenga vigencia. Ésta no es una situación en que podamos prescindir de la excepcionalidad. Las excepciones prácticamente son ilimitadas. Esto es lo que realmente entraña la indeterminación psicológica. La conspiración de las creencias, deseos y acciones harían imposible la tarea de especificación de las condiciones iniciales.

Sin embargo, se podría argumentar que las excepciones no ofrecen problema, porque no son azarosas. Las excepciones son causadas por factores que o bien cancelan, o bien compiten con el factor mencionado en la cláusula "si..." de la generalización original. Pueden haber factores cancelatorios para los que una generalización como la siguiente funciona: "Si e, entonces (ceteris paribus), A no conducirá a B".

Pero esto no modifica la situación. Aun cuando no fueran azarosas, no serían especificables. Supongamos que quisiéramos saber por qué Patricia fue a la librería a comprar un libro en especial. Ella quería un libro, y creía que podría conseguirlo en la librería, y no tenía una razón que le impidiera conseguirlo. Si usáramos una cláusula ceteris paribus tal como (L) no estaríamos realmente explicando por qué fue a la librería. Los factores en competencia son importantes en la explicación del comportamiento, puesto que podría ser que Patricia quisiera el libro, pero tam- 
bién puede ser cierto que ella quisiera otra cosa más con mayor intensidad. $\mathrm{O}$ supongamos que Patricia cree que el ir a la librería es la forma de obtener el libro, pero no la mejor, o más eficiente, o más barata. La lista podría continuar, pero el punto aquí es que con la imposibilidad de especificar los factores en competencia o cancelatorios, la ley ceteris paribus sería vacua.

Recordemos que la ley ceteris paribus lleva la cláusula que podemos interpretar como "no tenía otra razón mayor que interfiriera". Si especificamos las variables individuales de la ley ceteris paribus terminamos diciendo algo como: "Patricia fue a la librería a menos que no fue" (no tuvo otra razón mayor), lo que es patentemente poco informativo y vacuo. Y si la ley es vacua, es infalseable. Ninguna evidencia sobre la acción humana podría llevar a negar esta ley. Y una ley infalseable no puede proporcionar conocimiento empírico.

Aún más, se podría objetar argumentando contra la indeterminación y sostener que la imposibilidad de enunciar las condiciones iniciales es sólo un problema práctico. Después de todo, la psicología es una ciencia joven y con el tiempo podría llegar a una relativa precisión como se encuentra en las ciencias físicas. Esto parece razonable. Pero el objeto de estudio de la psicología es precisamente lo que impide la especificación no sólo por cuestiones prác- ticas, sino en principio ${ }^{4}$. Consideremos la creatividad como una característica del comportamiento humano. La manera como los seres humanos se adaptan y se comportan en formas novedosas pone en cuestionamiento cualquier relación determinista o legal. Nuestro autoconocimiento resulta en innovaciones que alteran significativamente nuestro comportamiento. Como una ilustración podemos ver hoy cómo la Revolución Francesa alteró las formas en que las personas percibían el mundo y las relaciones entre sí y sus instituciones.

Emergen nuevas formas de relaciones $\mathrm{y}$, por lo tanto, los individuos empiezan a comportarse en formas novedosas. En consecuencia, cualquier explicación legal, ceteris paribus o no, no sólo sería inapropiada, pero falsa. La creatividad pone en tela de juicio la noción tradicional de regularidad, y por ende, la necesidad nomológica. Como expresa Brian Fay comentando sobre el objeto de estudio de las ciencias sociales:

\begin{abstract}
... los objetos de la ciencia social son abiertos en una forma práctica impredecible. Las instituciones y prácticas, así como las creencias y deseos de los miembros de grupos sociales particulares, están continuamente en un estado de flujo y evolución, apareciendo inde-
\end{abstract}

4 La indeterminación psicológica no es comparable a la indeterminación cuántica, como podría pensarse, puesto que las leyes de la mecánica cuántica son estadísticas. 
terminado a aquellos que desean estudiarlos (1994, p. 103).

La búsqueda de leyes tendría sentido si hubiera un patrón que pudiera ser verificado de algún continuum o al menos un objeto que pudiera evolucionar en una manera predecible. Pero la lección que se aprende acerca de la creatividad humana es que hay una imposibilidad de confirmación. Los fenómenos a estudiarse serán eventualmente diferentes.

Un problema adicional, en el caso de la psicología, es el relacionado con el hecho de las múltiples realizaciones de los estados mentales. Gracias a Hilary Putnam (1992) estamos familiarizados con el tema de las realizaciones múltiples. Brevemente, la tesis de la realización múltiple sostiene que no habría un único tipo físico singular (¿neuronal?) que realizaría un estado mental particular; existiría una multiplicidad de tipos físicos que podrían realizar un estado mental particular. Por lo tanto, no habría una relación necesaria entre estar en un estado físico particular y estar en un estado mental particular. Esto no quiere decir que hay una independencia entre lo mental y lo físico. Sólo enfatiza las variedades de dependencia. Por esto es posible afirmar que la realizabilidad múltiple es compatible con la noción de superviniencia, puesto que un estado mental particular puede supervenir en diferentes condiciones físicas sin haber nada en común en el nivel físico (McGinn, 1991, p. 141).
La noción de ley ideal proviene de una concepción de la naturaleza resultado de la aplicación de un principio como "misma causa, entonces mismo efecto", denominado regularismo humeano (Hume, 1886). Este principio lleva a que se crea que, si uno asume que las leyes explican la conexión entre causas y efectos, sólo hay una explicación correcta para un fenómeno particular, cubierto por una ley. Pero nada podría estar más lejos de la práctica científica actual. Por ejemplo, en la física hay explicaciones teoréticas alternativas para el mismo fenómeno. Los físicos construyen diferentes modelos para diferentes propósitos utilizando diferentes ecuaciones para describir estos modelos (Cartwright, 1983, p. 11). Preguntar cuál es el modelo correcto o cuál es el "verdadero" conjunto de ecuaciones es perder de vista la metodología de la práctica científica. El punto es que un modelo particular "muestra algunos aspectos del fenómeno; un modelo diferente, otros" (Cartwright, 1983, p. 11). Lo que es más interesante es que diferentes enfoques teoréticos para explicar un fenómeno particular resultan en diferentes leyes para exactamente el mismo fenómeno (Cartwright, 1983, pp. 80-81).

Estas conclusiones sugieren una revisión de nuestro concepto tradicional de ley científica: no es ni universal ni está libre de excepciones. Pero si las leyes no son estrictas, entonces su rol en las explicaciones de cobertura legal es 
cuestionable. Colocar un fenómeno singular bajo la cobertura de una ley aparentemente sin excepciones llevaría a una situación paradójica: puesto que los fenómenos singulares fuera del contexto experimental no se comportan de la manera descrita por las leyes, resultaría que el fenómeno no tendría una explicación causal. No es que se afirme que las leyes no sean útiles. Podría ser el caso que sean falsedades útiles, pero lo que se propone es un desafío a la creencia que las leyes son componentes necesarios de cualquier explicación completa. Puede ser que las leyes sean utilizables en algunas explicaciones, si el enfoque teórico así lo requiriera, pero no existe una razón a priori para hacerlo. El modelo de cobertura legal asume que una ley "verdadera" hará el trabajo explicatorio, pero hemos visto cómo diferentes leyes pueden explicar un mismo fenómeno. Si no hay leyes verdaderas, y diferentes leyes pueden explicar un fenómeno, el rol de las leyes en el modelo de cobertura puede ser otro que el explicatorio.

\section{UN MODELO ALTERNATIVO:}

\section{EXPLICACIÓN NO-CAUSAL}

A pesar de los problemas que se derivan de la aplicación del modelo nomológico-deductivo, ha habido un tácito reconocimiento de que tal modelo es adecuado para las ciencias naturales, y que las ciencias humanas, incluida la psicología, deberían tener un método propio. Bajo esta perspectiva, existen diferencias irreconciliables entre la explicación de la naturaleza y la explicación de los seres humanos que demandan distinciones metodológicas y ontológicas entre ambas ciencias. Propulsores de esta visión (Taylor, 1980; 1985) comparten la convicción de que las ciencias humanas debieran ser más "interpretativas" que descriptivas. Puesto que los seres humanos son agentes autointerpretativos, sus acciones son significativas. Por lo tanto, el objetivo de las ciencias humanas no es adquirir objetividad como en las ciencias naturales sino comprender los significados de las acciones humanas.

De acuerdo con algunos investigadores, la psicología popular es una actividad interpretativa. Interpretar el comportamiento de alguien es atribuir actitudes a un individuo basado en lo que él/ella dice o hace. Pero esta atribución no es causal sino de significado. Cuando damos sentido al comportamiento de una persona atribuyéndole creencias y deseos, realizamos una forma de interpretación. Pero, como se vio arriba, tenemos la intuición de que las explicaciones de la psicología popular son una forma de explicación causal en que cuando se describe una acción como intencional, hacemos referencia a su historia causal. Si P fue a la tienda a conseguir vino, entonces el deseo de tener una botella de vino debe causalmente explicar, al menos parcialmente, la acción de $P$.

Podemos advertir que se crea una tensión entre dos formas de interpretacio- 
nismo: una que niega un elemento causal en la comprensión de la acción humana y la otra que es comprometida con la causalidad en la explicación de la acción. Pero es posible mostrar que el interpretacionismo no es incompatible con el causalismo en que, contrariamente a la percepción ordinaria, la interpretación no es una práctica exclusiva de las ciencias humanas sino que puede ser extendida al campo total del conocimiento humano.

Tomemos una de las convicciones de Taylor, la de que las ciencias humanas requieren la comprensión en el sentido hermenéutico. La oposición que Taylor construye entre las ciencias humanas y naturales es familiar para nosotros desde Dilthey (1976): las ciencias naturales explican, las humanas interpretan y comprenden. Pero, ¿es válida esta dicotomía?

Uno podría encontrar muchos ejemplos en las ciencias naturales en las que el término "comprensión" es usado adecuadamente. Sin embargo, Taylor notaría que "comprender textos" o "comprender motivos" es algo diferente a la "comprensión" que tiene lugar en las ciencias naturales. Pero eso no se sigue puesto que es verdad que un exégeta bíblico o una historiadora hacen el esfuerzo para comprender un pasaje en especial o una cultura particular, pero esto no constituye una indicación que no podríamos encontrar una analogía en la física o en la biología. Un paleontólogo tendría, en efecto, dificultad pa- ra comprender un resto fósil y colocarlo en un período geológico. $\mathrm{O}$ un matemático para comprender teoremas para usarlos en siguientes pruebas. Se podría contraargumentar esto diciendo que no hay nada hermenéutico en la comprensión empírica. El punto aquí, sin embargo, es que el término "comprensión" no contribuye a la justificación de la diferenciación radical que se pretende.

Lo mismo se aplicaría al término "explicación". No habría problema al asumir que proposiciones como "La Relatividad General explica más fenómenos que la mecánica newtoniana". Después de todo, de acuerdo con Taylor, se supone que las ciencias naturales deben explicar. Pero, ¿acaso no podríamos decir que un semiólogo explica los significados de ciertos símbolos de una cultura o que una psicóloga explica por qué un estudiante dejó el salón de clases luego de ser gritado por su profesora?

Otro punto problemático en esta visión es la casi ausencia de la noción de causalidad en el análisis de la acción humana. Aquí deben advertirse dos problemas.

Primero, no hay razón alguna a priori para sostener que la interpretación de la acción y el causalismo sean incompatibles. Consideremos el argumento de Davidson para la concepción causal de la acción (1980). Para Davidson las razones explican las acciones. S realizó esta acción porque tenía una razón particular. Ahora, este "porque" se refiere 
a la relación causal entre el haber una razón para la acción (causa) y la acción como resultado de esa razón (efecto). Es verdad que uno podría utilizar la palabra "porque" para referirse a la explicación no-causal, como cuando se me pregunta por qué la torre se mueve en línea recta en el tablero de ajedrez, por lo que esperaría no una explicación causal sino por qué tiene las propiedades que manifiesta o por qué sigue cierta regla en particular. Pero, como afirmé arriba, las explicaciones científicas tienen que hacer con explicar por qué una cosa ocurrió. Y eso, como Davidson arguye, es una pregunta que demanda una respuesta causal.

Se podría objetar esto último sosteniendo que lo que se busca en las ciencias humanas es la "comprensión" del significado o importancia de una acción, no por qué ocurrió. Cuando hablamos acerca de una acción tratamos de darle sentido cuando "hay una coherencia entre las acciones del agente y el significado de la situación para él/ella" (Taylor, 1985, p. 24). Y en el caso de una acción contradictoria o irracional, tratamos de entenderla, cuando comprendemos por qué se realizó. ¿Por qué el análisis de ambos casos -la búsqueda de significado del acto racional y del irracional- excluye una reconstrucción etiológica? Davidson aporta una luz a esta pregunta: "Los eventos son frecuentemente descritos en términos de sus causas. Supongamos que alguien fue herido. Podríamos redescribir el evento 'en términos de causas' diciendo que fue quemado" (1980, p. 10). Aun cuando el significado de la acción conlleva una connotación moral (valor) o legal, esto no previene una reconstrucción en términos causales que es precisamente lo que la explicación supone que debe proporcionar. Lo mismo puede decirse de la significación de las recientes teorías del caos en los asuntos humanos. La teoría del caos es una herramienta interesante y poderosa para explicar fenómenos pero también puede ser una fuente de interpretación significativa en las vidas de las personas. Necesitamos recordar que el objetivo de una buena explicación es la comprensión de la metafísica involucrada en un evento. El empleo de la causación no es arbitrario, sino uno que hace más inteligible por qué ocurren cosas, y en el caso de la acción, por qué las personas se comportan de la manera que lo hacen. En este sentido, el nexo metafísico entre razón y acción, como defiende Davidson, se comprende mejor como una relación causal. Parte de la razón por la que debería haber una comprensión causal de la acción humana proviene de la idea que tenemos acerca del mundo. Una comprensión causal de la acción calza metafísicamente con el resto del mundo físico en el cual las ocurrencias causales son comunes.

Segundo, tenemos el asunto sobre el origen de los conceptos. En las ciencias naturales podemos ver cómo muchos 
de los conceptos están relacionados con su origen causal. Considérese el siguiente significado de "glucógeno". El glucógeno es un polvo blanco insípido, soluble en agua, pero forma, como el almidón, una solución opalescente. Es insoluble en alcohol y éter. Es dextrorotatorio. Con el test de Trommer da una solución azul, pero no ocurre reducción cuando hierve. Del mismo modo, una cantidad de conceptos se derivan de sus propiedades causales. El oxígeno es, por definición, un gas que sostiene la combustión; el hidrógeno es, por definición, un elemento cuya combustión resulta en vapor de agua; el nitrógeno es, por definición, capaz de matar animales cuando se le aspira en forma pura (Hanson, 1969, p. 39). Estas definiciones pueden ayudar a explicar lo que los elementos significan para químicos o biólogos.

Por analogía, algunos de los significados de las acepciones usadas por Taylor pueden ser definidas en términos causales. Tomemos un ejemplo del mismo Taylor, "vergüenza":

Un término como "vergüenza", por ejemplo, se refiere esencialmente a cierto tipo de situación, lo "vergonzoso", o "humillante", y a una cierta forma de respuesta, aquella de esconderse, cubrirse... Esto es esencial a la identificación del sentimiento como vergüenza que está relacionado con esta situación y que da lugar a este tipo de disposición. Pero esta situación a su vez puede sólo ser identificada en relación a los sentimientos que provoca $(1985$, p. 23$)$.
Fácilmente podemos ver cómo este pasaje hace una aseveración causal implícita. La vergüenza es el resultado de lo vergonzoso o humillante. Como resultado del evento humillante, el agente es causado a esconderse o cubrirse. Y no debería sorprendernos, puesto que Taylor utiliza categorías y explicaciones de la psicología popular que, como vengo defendiendo, utiliza una metafísica causal.

\section{HACIA UNA TEORÍA SINGULAR DE LA EXPLICACIÓN}

Finalmente, se esbozarán algunas ideas y posibles problemas para la formulación de una teoría de la explicación singular. Si se encuentran problemas en los dos modelos mencionados, podríamos introducir una forma alternativa de teoría de la explicación que considero que puede ser más adecuada en las explicaciones psicológicas. Si damos lugar a una concepción más amplia de la explicación que consiste en la descripción de las causas subyacentes que provocan un fenómeno (Miller, 1987) podríamos proporcionar una explicación satisfactoria sin requerir leyes psicológicas. Las explicaciones deberían captar las relaciones causales, por lo tanto, es esencial que las explicaciones genuinas identifiquen la relación entre causa y efecto. El tipo de explicación que tengo en mente funciona a través de proposiciones casuales singulares. Si, como sospecho, hay poca esperanza para la formulación de le- 
yes psicológicas, entonces un modelo de explicación causal singular puede ser más promisorio. En este modelo, la naturaleza de su poder explicativo es clara: explica no porque hay una ley o una proposición con condiciones suficientes, sino porque identifica las condiciones tales, que cambios en éstas harían una diferencia en el fenómeno a ser explicado (Woodward, 1984).

La explicación singular causal tiene al menos dos restricciones que podrían verse desde un ángulo positivo o negativo: el desafío a la visión de la causación de Hume (regularidades) y la admisión de que no todas las explicaciones tendrán capacidad predictiva. Pero las explicaciones causales singulares hacen lo requerido en cualquier explicación causal: especifica el evento que causó el efecto descrito en el explanandum. Además, permite la postulación de contrafácticos: si el evento en el explanans no hubiera ocurrido, entonces las cosas hubieran sido diferentes. Esto ocurre porque la instancia de una propiedad (v.g., deseo de beber) es causalmente eficaz. Si no hubiera ocurrido esa instancia, el evento explicado (v.g., levantar el brazo para alcanzar el agua) no hubiera ocurrido. La explicación causal singular sigue a la explicación científica general, puesto que es siempre una explicación de por qué el resultado dado ocurrió a diferencia de resultados posibles alternativos. Tales explicaciones proceden mostrando que dadas las condiciones, el resultado fue preferido sobre las alternativas.
Por otro lado, como se dijo anteriormente, el mundo, tal como se presenta, no muestra las regularidades que preferirían los teóricos universalistas de la causación. Suponiendo que los regularistas humeanos sean conscientes de que vivimos en un universo indeterminado, encontrarían aún deseable reducir proposiciones singulares en proposiciones genéricas. Esto se puede lograr a través de diferentes estrategias. Una de ellas es la de obtener promedios. Para ello se requiere concentrarse sólo en las similitudes que tienen los objetos entre sí. Cualquier elemento disímil que podría convertirse en un factor causal e influir en el resultado causal quedaría descartado. Consideremos el siguiente ejemplo:

A un ratón de laboratorio se le adminis-
tra una dieta conteniendo tanto giromi-
trina y un metabolito del diazonio y
contrae un tumor estomacal. Ninguna
sustancia es suficiente para producir el
tumor en dosis pequeña, pero cada una
individualmente ha demostrado que in-
crementa la incidencia de tumores en
los ratones de laboratorio (Humphreys,
1989, p. 15).

¿Cuál fue la causa del tumor? ¿La primera o la segunda sustancia? ¿O ambas? La incidencia en una población promedio nos da alguna información pero no ayuda en ocasiones como ésta. Como resultado de un promedio, la segunda sustancia puede ser considerada como "ruido" y tomada como irrelevante. Sin embargo, el promediar no ayudará a encontrar el rol de la segun- 
da sustancia. La clave para entender el fenómeno en el ejemplo es incluir la causación singular en juego y estudiar los factores implicados en este caso individual. El promediar esconderá inevitablemente aquellas fuerzas relevantes. Lo mismo y en grado mayor de complejidad se podría inferir para el estudio de la conducta humana.

Mi impresión es que un modelo de explicación causal singular evita algunas de las deficiencias del modelo hempeliano. Sin embargo, uno de los asuntos que necesita ser más analizado es el perenne problema del epifenomenalismo. De acuerdo con la objeción del epifenomenalismo, aún las explicaciones causales singulares pueden ser culpadas de ser redundantes. La redundancia aparece como resultado de la naturaleza omniabarcante de la teoría física. Reconfortarse con un instrumentalismo al modo de Dennett (1987) no serviría, puesto que la explicación de la psicología popular sería simplemente un paradero en el camino a la explicación fisicalista final que vendrá, pero inaccesible temporalmente por nuestra ignorancia actual. Para este problema quizás no deberíamos buscar las respuestas tanto en la psicología misma, sino en otras ciencias, como la biología, que defiende una independencia de la teoría física. Hemos visto cómo enfoques reduccionistas en biología no han llegado muy lejos (motivados quizás por el éxito de la biología molecular) y no tan lejos, de hecho, como el sugerir que los términos como "célula", "especie", y otros parecidos son simples epifenómenos de los quarks y las fuerzas. Pero esto requiere más investigación.

\section{REFERENCIAS}

Block, N. (Ed.)(1980). Readings in philosophy of psychology. Vol. 1. Cambridge, MA.: Harvard University Press.

Boden, M. (1972). Purposive explanation in psychology. Cambridge, MA: Harvard University Press.

Cartwright, N. (1983). How the laws of physics lie. New York: Oxford University Press.

Child, W. (1994). Causality, interpretation and the mind. Oxford: Clarendon Press.

Davidson, D. (1980). Essays on actions and events. Oxford: Clarendon Press.

Dennett, D. (1987). The intentional stance. Cambridge, MA.: MIT.

Dilthey, W. (1976). Selected writings. Cambridge: Cambridge University Press.

Fay, B. (1994). General laws and explaining human behavior. En M. Martin \& L. McIntyre. Readings in the philosophy of social science. Cambridge, MA.: MIT Press.

Feynman, R. (1965). The character of physical law. Cambridge, MA: MIT.

Fodor, J. (1975). The language of thought. Hassocks: Harvester Press.

Fodor, J. (1987). Psychosemantics. Cambridge, MA.: MIT. 
Garfinkel, A. (1981). Forms of explanation: Rethinking the questions in social theory. New Haven: Yale UP.

Guttenplan, S. (Ed.)(1995). A companion to the philosophy of mind. Oxford: Blackwell.

Hanson, N. (1969). Perception and discovery: An introduction to scientific inquiry. San Francisco: Freeman, Cooper and Company.

Hawking, S. (1988). A brief history of time. New York: Bantam Books.

Hempel, C. (1965). Aspects of scientific explanation and other essays in the philosophy of science. New York: The Free Press.

Horgan, T. y Tienson, J. (1990). Soft laws. En P. French (Ed.). The philosophy of the human sciences. Notre Dame: University of Notre Dame Press.

Hume, D. (1886/1964). A treatise of human nature. Darmstadt: Scientia Verlag Aalen.

Humphreys, P. (1989). Scientific explanation: The causes, some of the causes, and nothing but the causes. En P. Kitcher W. Salmon (Eds.). Scientific explanation. Minneapolis: University of Minnesota Press.

Kim, J. (1993). Supervenience and mind: selected philosophical essays. Cambridge: Cambridge University Press.

McGinn, C. (1991). The problem of consciousness: Essays towards a resolution. Oxford: Basil Blackwell.

Miller, R. (1987). Fact and method: Explanation, confirmation and reality in the natural sciences. Princeton, N.J.: Princeton University Press.
Osherson, D. (1995). An invitation to cognitive science. Cambridge, MA.: MIT.

Putnam, H. (1992). The Nature of Mental States. En B. Beackley \& P. Ludlow (Eds.). The philosophy of mind. Cambridge, MA: MIT.

Rosenberg, A. (1988). Philosophy of social science. Oxford: Clarendon Press.

Taylor, C. (1980). "Understanding in human science". Review of Metaphysics, 34, 25-38.

Taylor, C. (1985). Interpretation and the sciences of man. Philosophy and the human sciences: Philosophical Papers. Vol. 2. Cambridge: Cambridge University Press.

von Wright, G. (1971). Explanation and understanding. Ithaca, NY: Cornell University Press.

Woodward, J. (1984). A theory of singular causal explanation. Erkenntnis, 21, 231-262. 